\title{
Association between the PLTP rs4810479 SNP and Serum Lipid Traits in the Chinese Maonan and Han Populations
}

\author{
Fen-Han Zhang, ${ }^{1}$ Rui-Xing Yin $\mathbb{D}^{1}{ }^{1}$ Li-Mei Yao, ${ }^{1}$ Wei-Xiong Lin, ${ }^{2}$ Jin-Zhen Wu, ${ }^{1}$ \\ and De-Zhai Yang \\ ${ }^{1}$ Department of Cardiology, Institute of Cardiovascular Diseases, The First Affiliated Hospital, Guangxi Medical University, \\ Nanning, Guangxi, China \\ ${ }^{2}$ Department of Molecular Genetics, Medical Scientific Research Center, Guangxi Medical University, Nanning, Guangxi, China
}

Correspondence should be addressed to Rui-Xing Yin; yinruixing@163.com

Received 24 March 2021; Accepted 22 June 2021; Published 3 July 2021

Academic Editor: Chaeyoung Lee

Copyright ( $) 2021$ Fen-Han Zhang et al. This is an open access article distributed under the Creative Commons Attribution License, which permits unrestricted use, distribution, and reproduction in any medium, provided the original work is properly cited.

\begin{abstract}
The association between the phospholipid transfer protein (PLTP) gene rs4810479 single-nucleotide polymorphism (SNP) and serum lipid levels is largely unknown. This investigation aimed to evaluate the relationship between the PLTP rs4810479 SNP, several environmental risk factors, and serum lipid parameters in the Chinese Maonan and Han nationalities. Polymerase chain reaction-restriction fragment length polymorphism, gel electrophoresis, and direct sequencing were employed to determine the PLTP rs4810479 genotypes in 633 Maonan and 646 Han participants. The frequencies of CC, CT, and TT genotypes and the C allele were different between Maonan and Han groups (29.07\%, 53.08\%, 17.85\%, and 55.61\% vs. 35.60\%, 49.70\%, $14.70 \%$, and $60.45 \%$, respectively, $P<0.05)$. The $\mathrm{C}$ allele carriers in the Maonan group had higher high-density lipoprotein cholesterol levels than the $\mathrm{C}$ allele noncarriers, but this finding was only found in Maonan males but not in females. The $\mathrm{C}$ allele carriers in Han males had lower total cholesterol and low-density lipoprotein cholesterol levels than the $C$ allele noncarriers. Serum lipid profiles were also affected by several traditional cardiovascular risk factors in both populations. There might be an ethnic- and/or sexspecific association between the PLTP rs4810479 SNP and serum lipid traits.
\end{abstract}

\section{Introduction}

Cardiovascular disease (CVD) is one of the leading causes of disability and early death worldwide, accounting for about one-third of the global mortality rate [1]. The cost of CVD constitutes a major economic burden to the society [2]. Many studies have proven that serum or plasma triglyceride (TG), low-density lipoprotein cholesterol (LDL-C), and high-density lipoprotein cholesterol (HDL-C) concentrations are independent risk factors for CVD [3-5].

It is well known that various genetic and environmental factors can lead to abnormalities of plasma lipids and lipoproteins [6-8]. Plasma lipid and lipoprotein concentrations are themselves highly heritable-estimates range from $40 \%$ to $60 \%$. A number of genome-wide association studies (GWASes) have identified more than 95 genetic loci associated with plasma lipid phenotypes. One of the newly discovered loci is the phospholipid transfer protein (PLTP) gene [9-12].

PLTP (also called lipid transfer protein 2) is a member of lipid transfer/lipopolysaccharide- (LPS-) binding protein family. This family includes PLTP, LPS-binding protein (LBP), bactericidal/permeability-increasing protein (BPI), and cholesterol ester transfer protein (CETP) [13-15]. There are two molecular weights of PLTP, $55 \mathrm{kDa}$ and $81 \mathrm{kDa}$. This may be due to different glycosylation [15]. PLTP is a monomeric and nonspecific lipid transfer protein, which can efficiently transfer free cholesterol, diacylglycerol, $\alpha$-tocopherol, cerebroside, LPS, phospholipids, and sphingosine1-phosphate [13-15]. There are two forms of lipoproteinassociated plasma PLTP (high active one and low active one) which are associated with apolipoprotein (Apo) A1- and 
ApoE-containing lipoproteins, respectively [16]. However, the cause for the existence of active and inactive PLTP in plasma is unclear. It is quite possible that PLTP might have other activities except its lipid transfer function [15]. PLTP is produced in various types of cells and secreted into plasma. It is highly expressed in human tissues such as the ovary, thymus, placenta, lung [17], liver, and small intestine and in macrophages $[18,19]$ and atherosclerotic lesions $[19,20]$. The gene encoding PLTP (PLTP) is located on human chromosome 20. Its cDNA has a length of 1750 base pairs, including an open reading frame of 1518 nucleotides and a 3 '-untranslated region (UTR) of 184 nucleotides. The mature PLTP contains 476 amino acids and $6 \mathrm{~N}$-glycosylation sites that allow it to change its molecular weight (55 or $81 \mathrm{kDa}$ ) after different degrees of glycosylation modification [17]. Several previous studies have found that PLTP is an emerging cardiac metabolic factor which exerts a vital part in the development of blood lipid metabolism and atherosclerosis $[21,22]$. PLTP is a main factor modulating the size and composition of high-density lipoprotein particles in the circulation and plays an important role in controlling plasma HDL-C levels [23]. PLTP deficiency in mice can lower total cholesterol (TC), HDL-C, and ApoA1 but increase TG levels significantly, impact the biological quality of high-density lipoprotein [24], and attenuate high-fat dietinduced insulin resistance and obesity [25]. Plasma PLTP activity (PLTPa) was significantly inversely correlated with carotid artery disease (CAAD), with a $9 \%$ decrease in odds of CAAD per 1 unit increase in PLTPa. Plasma TG levels, diabetes, statin use, and PLTP rs4810479 SNP were also associated with PLTPa significantly [26]. In human studies, both PLTP mass and PLTPa were associated with plasma lipid traits, glucose regulation, and atherosclerosis. Common variation at the PLTP structural locus region could explain about $30 \%$ of variation in PLTPa [27]. PLTP variants were associated with the PLTP mRNA level [28] and CVD risk [27, 29].

Being an isolated and conservative minority in China, the population of Maonan nationality was 107,166 (ranked 37) according to the statistics of China's sixth national census in 2010. They have own unique culture and life customs, such as intraethnic marriages, clothing, special lifestyle, and dietary structure. These characteristics are distinct from those in the largest ethnic group, Han Chinese. Therefore, we hypothesize that the genotype distribution and genetic traits of some lipid metabolism-related genes in the Maonan ethnic group may be different from those in the Han ethnic group. In the Chinese populations, there is no previous study to explore the association between the PLTP rs4810479 SNP and serum lipid levels. Thus, the aim of this study was to appraise the association between the PLTP rs4810479 SNP, several environmental risk factors, and serum lipid traits in the Maonan and Han nationalities.

\section{Materials and Methods}

2.1. Subjects. The study populations were stochastically chosen from our earlier stratified random specimens. The detailed inclusion and exclusion criteria have been described in a previous report [30]. In brief, all selected people were basically healthy and had no evidence of any chronic illness such as cardiac, hepatic, renal, or thyroid diseases. The participants who had a history of heart attack or myocardial infarction, stroke, congestive heart failure, and diabetes were excluded. They did not use medications known to affect serum lipid levels such as lipid-lowering drugs (statins or fibrates), $\beta$-blockers, diuretics, or hormones. The present study included 633 unrelated Maonan participants (251 males, $39.65 \%$, and 382 females, $60.35 \%$ ) and 646 unrelated Han subjects (268 males, $41.49 \%$, and 378 females, $58.51 \%$ ) [30]. The participants aged from 22 to 92 years (mean: $55.92 \pm 14.30$ years in Maonan and $54.50 \pm 14.50$ years in Han groups). The age structure and sex ratio between the two populations were matched. Basic information and health status of all participants refer to our previous study [31]. This research project was approved by the Ethics Committee of the First Affiliated Hospital, Guangxi Medical University (no. Lunshen-2014-KY-Guoji-001, March 7, 2014). All participants provided written informed consent before the study.

2.2. Epidemiological Survey. The survey was conducted using an internationally standardized method [32]. A standardized questionnaire was used to gather the information related to demographic statistics, socioeconomic status, and life style factors. Drinking and smoking were grouped according to daily consumption $(0, \leq 25$, and $>25$ and $0, \leq 20$, and $>20$, respectively). Several parameters such as weight, body mass index (BMI), height, waist circumference, and blood pressure were also obtained.

2.3. Biochemical Measurements. After 12 hours of fasting, a cubital vein blood sample of $5 \mathrm{ml}$ was obtained from all participants. Biochemical measurements including TC, TG, HDL-C, LDL-C, ApoA1, ApoB, and blood glucose were performed as previously described $[33,34]$.

2.4. DNA Amplification and Genotyping. Genomic DNA of the samples was extracted by the phenol-chloroform method [34]. Polymerase chain reaction-restriction fragment length polymorphism (PCR-RFLP) was utilized to determine the genotypes of the PLTP rs4810479 SNP. The forward and reverse primer pairs for PCR amplification were $5^{\prime}$ ATCCTCCGATCTTGGCTTCC- $3^{\prime}$ and $5^{\prime}$-CCAGGTAGAGGGAACAGCAA- $3^{\prime}$, respectively. The specific reaction condition was $5 \mathrm{~min}$ pretreatment at $95^{\circ} \mathrm{C}$, denaturation at $95^{\circ} \mathrm{C}$ for $30 \mathrm{~s}$, annealing at $59^{\circ} \mathrm{C}$ for $30 \mathrm{~s}$, followed by extension for $40 \mathrm{~s}$ at $72^{\circ} \mathrm{C}$ for 33 cycles, and finally a $7 \mathrm{~min}$ extension at $72^{\circ} \mathrm{C}$. The restriction enzyme was KpnI. After electrophoresis on $2.0 \%$ agarose gel containing $0.5 \mu \mathrm{g} / \mathrm{ml}$ of ethidium bromide, the results were obtained under ultraviolet light. The PCR products of six samples were also confirmed by direct sequencing using ABI Prism 3100 (Applied Biosystems) in Shanghai Sangon Biological Engineering Technology \& Services Co., Ltd., China. 
2.5. Diagnostic Criteria. The normal reference values of serum lipid parameters including TG, TC, LDL-C, HDL-C, ApoA1, ApoB concentrations, and $A p o A 1 / A p o B$ ratio as well as the diagnostic criteria of hyperlipidemia, hypertension, and type 2 diabetes, and normal weight, overweight, and obesity have been described in detail in our several previous research studies [30, 31, 33-35].

2.6. Statistical Analysis. The number of study samples in this research was estimated using Quanto software. All of the statistical analyses were accomplished using SPSS software (version 23.0). Normally distributed quantitative variables were expressed as mean \pm standard deviation (nonnormally distributed serum TG levels were expressed as median and quartiles). Direct counting and standard goodness-of-fit test were used to determine the allele frequency and verify the Hardy-Weinberg equilibrium (HWE), respectively. The genotype distribution was tested by chi-square test, and the general features between the two ethnic groups were analyzed by unpaired $t$-test. The association between genotype and serum lipid parameters was assessed by the covariance analysis (ANCOVA), in which age, gender, blood pressure, BMI, cigarette smoking, and alcohol consumption were used as covariates. Stepwise modeling of multiple linear regression analyses was used to determine the relevant risk factors for serum lipid parameters in the Maonan, Han, male, and female (CC/CT genotypes $=1$ and TT genotype $=2$ ), respectively. Bilateral $P$ value $<0.05$ was considered statistically significant.

\section{Results}

3.1. General Features and Serum Lipid Profiles. The features and serum lipid levels are presented in Table 1 . The values of gender ratio, age structure, BMI, weight, and height; the percentages of cigarette smoking and alcohol intake; the levels of blood glucose, TC, LDL-C, ApoA1, and ApoB; and the ratio of $A$ poA1/ApoB were not different between the Maonan and Han populations $(P>0.05$ for all). However, the levels of waist circumference, pulse pressure, diastolic and systolic blood pressures, and serum TG were higher, whereas the levels of HDL-C were lower in Maonan than in Han ethnic groups $(P<0.05-0.001)$.

3.2. Genotyping and Genotypes. After electrophoresis of the PCR product, the products of $609 \mathrm{bp}$ nucleotide sequences were observed in all samples (Figure 1). The bands of the three genotypes are presented in Figure 2: CT genotype (286, 323, and $609 \mathrm{bp}$ ), CC genotype (286 and $323 \mathrm{bp}$ ), and TT genotype $(609 \mathrm{bp})$. The genotypes were distinguished by the presence of the enzyme restriction site ( $\mathrm{C}$ allele) or absence (T allele). The results of direct sequencing of the samples are shown in Figure 3.

3.3. Genotype and Allele Frequencies. As shown in Table 2, there were significant differences in the frequencies of CC, $\mathrm{CT}$, and TT genotypes and $\mathrm{C}$ allele between the Maonan and
Han populations $(29.07 \%, 53.08 \%, 17.85 \%$, and $55.61 \%$ vs. $35.60 \%, 49.70 \%, 14.70 \%$, and $60.45 \%$, respectively, $P<0.05)$. However, the genotype and allele frequencies of the rs4810479 SNP in both ethnic groups were not significantly different between men and women $(P>0.05$ for all).

3.4. Genotypes and Serum Lipid Concentrations. As summarized in Tables 3 and 4, serum HDL-C concentrations in the Maonan group were significantly different among the three genotypes $(P<0.05)$, and serum HDL-C concentrations were higher in the $\mathrm{C}$ allele carriers than the $\mathrm{C}$ allele noncarriers, but this finding was only restricted to males but not females. Lower TC and LDL-C concentrations in Han males were also observed in the $\mathrm{C}$ allele carriers than the $\mathrm{C}$ allele noncarriers $(P<0.05$ for all).

3.5. Relevant Factors for Serum Lipid Parameters. Multiple linear regression analyses showed that serum HDL$\mathrm{C}$ and ApoA1 concentrations in the Maonan group were correlated with the PLTP rs4810479 genotypes $(P<0.05$; Table 5). Serum HDL-C concentrations in Maonan males, HDL-C and ApoA1 concentrations in Maonan females, and TC and LDL-C concentrations in Han males were associated with the genotypes $(P<0.05$; Table 6$)$. In addition to the PLTP rs4810479 genotypes, serum lipid traits in the participants were also influenced by several risk factors such as gender, age, waist circumference, BMI, pulse pressure, diastolic blood pressure, systolic blood pressure, fasting blood glucose, alcohol consumption, and cigarette smoking $(P<0.05$ for all; Tables 5 and 6$)$.

\section{Discussion}

The current study revealed that the Maonan ethnic group had higher TG and lower HDL-C concentrations than the Han ethnic group $(P<0.001$ for each). There were no significant differences in the TC, LDL-C, ApoA1, and ApoB concentrations and the $A p o A 1 / A p o B$ ratio between the Maonan and Han populations $(P>0.05$ for all). It is common knowledge that dyslipidemia is one of the major changeable cardiovascular risk factors and is a major predictor of CVD mortality [1]. The difference in serum lipid profiles between the two populations may be due to distinct environmental, genetic factors and their interactions. Maonan nationality is one of 55 minorities in China. Being a mountain ethnic group, Maonan has its own unique history, custom, and culture, such as intraethnic marriages, specific clothing, inimitable lifestyle, and dietary habits. Most Maonan people are engaged in agricultural production, supplemented by animal husbandry, aquaculture, and other sideline industries. Rice and corn are their staple food, and pumpkin, sweet potato, and millet are the complementary foods. The preference for acidic food is the greatest feature of their diet culture. They have unique eating habits and lifestyles compared to other ethnic groups. Maonan ethnic group advocates intraethnic marriages. Their marriages are mostly arranged by parents. These results suggest that the 
TABle 1: Comparison of demographic, lifestyle characteristics, and serum lipid levels between the Maonan and Han populations.

\begin{tabular}{|c|c|c|c|c|}
\hline Parameter & Maonan & Han & $t\left(\chi^{2}\right)$ & $P$ \\
\hline Number & 633 & 646 & & \\
\hline Male/female & $251 / 382$ & $268 / 378$ & 0.446 & 0.531 \\
\hline Age (years) & $55.92 \pm 14.30$ & $54.50 \pm 14.50$ & 1.761 & 0.079 \\
\hline Height $(\mathrm{cm})$ & $154.09 \pm 8.22$ & $154.88 \pm 7.82$ & -1.756 & 0.079 \\
\hline Weight (kg) & $53.58 \pm 10.60$ & $53.32 \pm 8.88$ & 0.459 & 0.646 \\
\hline Body mass index $\left(\mathrm{kg} / \mathrm{m}^{2}\right)$ & $22.46 \pm 3.62$ & $22.20 \pm 3.21$ & 1.372 & 0.170 \\
\hline Waist circumference $(\mathrm{cm})$ & $76.73 \pm 9.04$ & $75.09 \pm 8.05$ & 3.415 & 0.001 \\
\hline \multicolumn{5}{|l|}{ Smoking status $(n(\%))$} \\
\hline Nonsmoker & $500(79.0)$ & $484(74.9)$ & \multirow{3}{*}{3.103} & \multirow{3}{*}{0.212} \\
\hline$\leq 20$ cigarettes/day & $116(18.3)$ & $139(21.5)$ & & \\
\hline$>20$ cigarettes/day & $17(2.7)$ & $23(3.6)$ & & \\
\hline \multicolumn{5}{|l|}{ Alcohol consumption $(n(\%))$} \\
\hline Nondrinker & $499(78.8)$ & $523(81.0)$ & \multirow{3}{*}{1.547} & \multirow{3}{*}{0.461} \\
\hline$\leq 25 \mathrm{~g} /$ day & $71(11.2)$ & $59(9.1)$ & & \\
\hline$>25$ g/day & $63(10.0)$ & $64(9.9)$ & & \\
\hline Systolic blood pressure (mmHg) & $134.49 \pm 23.29$ & $130.02 \pm 19.73$ & 3.697 & $<0.001$ \\
\hline Diastolic blood pressure $(\mathrm{mmHg})$ & $83.29 \pm 11.99$ & $81.55 \pm 11.01$ & 2.698 & 0.007 \\
\hline Pulse pressure $(\mathrm{mmHg})$ & $51.20 \pm 16.84$ & $48.47 \pm 15.77$ & 2.989 & 0.003 \\
\hline Glucose $(\mathrm{mmol} / \mathrm{L})$ & $6.08 \pm 1.25$ & $6.22 \pm 1.33$ & -1.934 & 0.053 \\
\hline Total cholesterol (mmol/L) & $4.99 \pm 0.97$ & $4.90 \pm 0.93$ & 1.640 & 0.101 \\
\hline Triglyceride $(\mathrm{mmol} / \mathrm{L})$ & $1.28(0.88)$ & $1.10(0.65)$ & 4.750 & $<0.001$ \\
\hline $\mathrm{HDL}-\mathrm{C}(\mathrm{mmol} / \mathrm{L})$ & $1.62 \pm 0.39$ & $1.83 \pm 0.42$ & -9.207 & $<0.001$ \\
\hline LDL-C (mmol/L) & $2.89 \pm 0.81$ & $2.84 \pm 0.70$ & 0.991 & 0.322 \\
\hline ApoA1 (g/L) & $1.39 \pm 0.23$ & $1.38 \pm 0.23$ & 1.165 & 0.244 \\
\hline ApoB $(g / L)$ & $0.88 \pm 0.19$ & $0.87 \pm 0.20$ & 1.032 & 0.302 \\
\hline ApoA1/ApoB & $1.66 \pm 0.50$ & $1.66 \pm 0.45$ & 0.013 & 0.990 \\
\hline
\end{tabular}

HDL-C: high-density lipoprotein cholesterol; LDL-C: low-density lipoprotein cholesterol; Apo: apolipoprotein. The value of triglyceride was presented as median (interquartile range); the difference between the two ethnic groups was determined by the Wilcoxon-Mann-Whitney test.

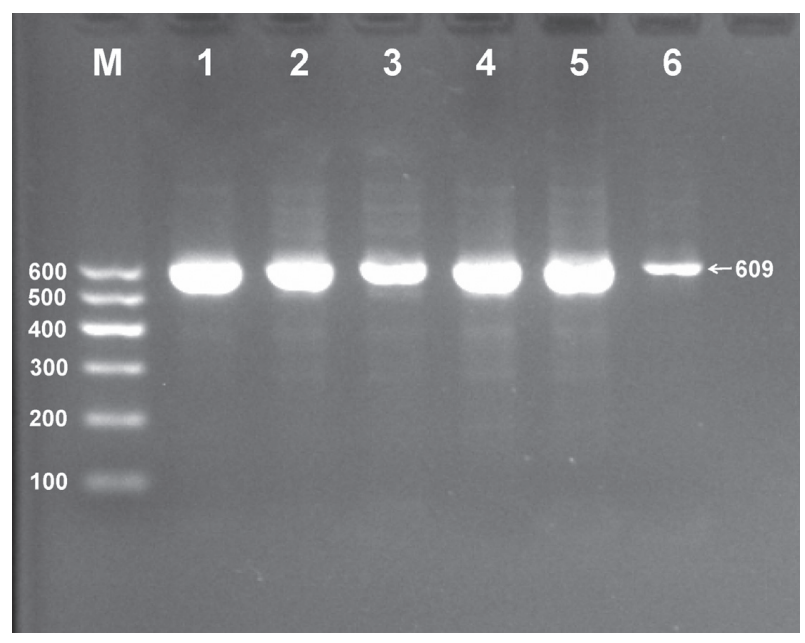

FIGURE 1: Electrophoresis of polymerase chain reaction products of the samples. Lane $\mathrm{M}$ is the $100-600 \mathrm{bp}$ marker ladder; lanes 1-6 are samples; the $609 \mathrm{bp}$ bands are the target genes.

genetic traits of some genes related to lipid metabolism may be different between the Maonan and Han ethnic groups.

According to the results of the International $1000 \mathrm{Ge}$ nomes database (https://www.ncbi.nlm.nih.gov/variation/ tools/1000genomes/), we knew that the rs4810479C allele frequency was $26.37 \%$ in British in England and Scotland (GBR); $27.27 \%$ in Utah residents (CEPH) with Northern and

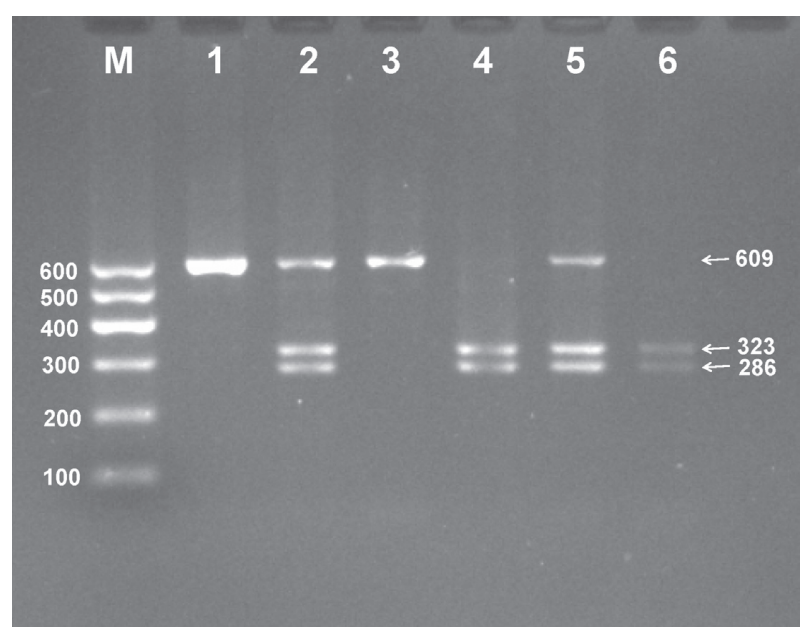

Figure 2: Genotyping of the PLTP rs4810479 SNP. Lane M, 100-600 bp marker ladder; lanes 1 and 3, TT genotype (609 bp); lanes 4 and 6, CC genotype (323 and $286 \mathrm{bp}$ ); lanes 2 and 5, CT genotype $(609,323$, and $286 \mathrm{bp})$.

Western European Ancestry (CEU); $32.45 \%$ in Colombians from Medellin, Colombia (CLM); $33.84 \%$ in Finnish in Finland (FIN); $41.67 \%$ in African Caribbean individuals in Barbados (ACB); $42.62 \%$ in Americans of African Ancestry in the southwestern USA (ASW); 43.43\% in Esan in Nigeria (ESN); $51.46 \%$ in Gujarati Indian from Houston, Texas 


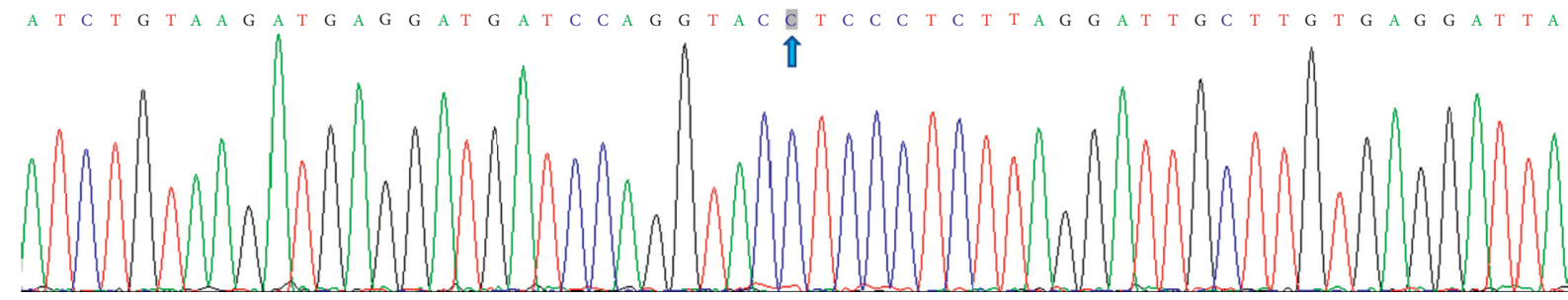

(a)

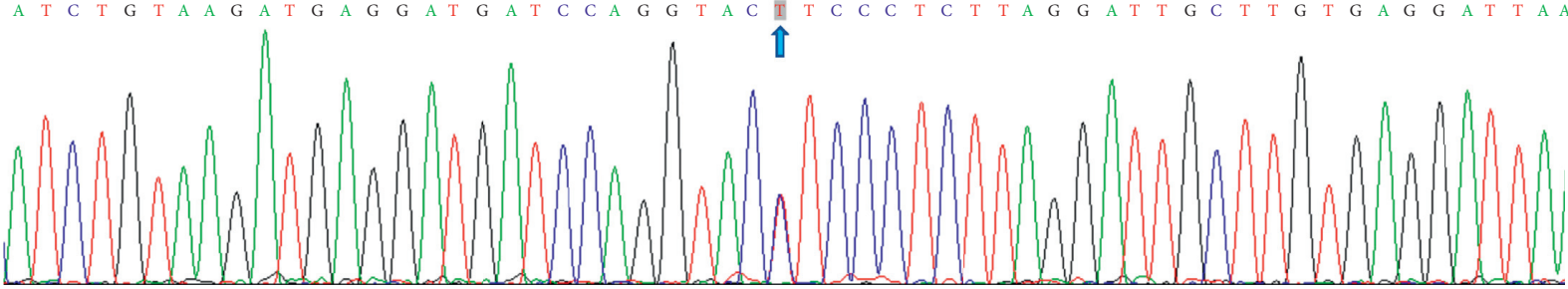

(b)

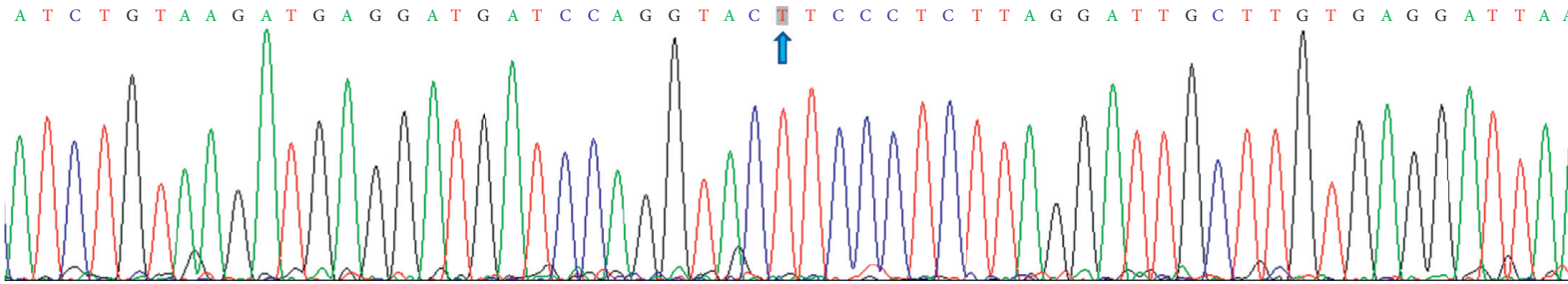

(c)

Figure 3: A part of the nucleotide sequence of the PLTP rs4810479 SNP: (a) CC genotype; (b) CT genotype; (c) TT genotype.

TABLE 2: Comparison of the genotype and allele frequencies of the PLTP rs4810479 SNP in the Maonan and Han populations ( $n$ (\%)).

\begin{tabular}{|c|c|c|c|c|c|c|c|}
\hline \multirow{2}{*}{ Group } & \multirow{2}{*}{$n$} & \multicolumn{3}{|c|}{ Genotype } & \multicolumn{2}{|c|}{ Allele } & \multirow{2}{*}{$P_{\mathrm{HWE}}$} \\
\hline & & $\mathrm{CC}$ & CT & $\mathrm{TT}$ & $\mathrm{C}$ & $\mathrm{T}$ & \\
\hline Maonan & 633 & $184(29.07)$ & $336(53.08)$ & $113(17.85)$ & $704(55.61)$ & $562(44.39)$ & 0.059 \\
\hline Han & 646 & $230(35.60)$ & $321(49.70)$ & $95(14.70)$ & $781(60.45)$ & $511(39.55)$ & 0.319 \\
\hline$\chi^{2}$ & & & 6.880 & & & & \\
\hline$P$ & & & 0.032 & & & & \\
\hline \multicolumn{8}{|l|}{ Maonan } \\
\hline Male & 251 & $78(31.08)$ & $128(51.00)$ & $45(17.93)$ & $284(56.57)$ & $218(43.43)$ & 0.549 \\
\hline Female & 382 & $106(27.79)$ & $208(54.29)$ & $68(17.92)$ & $420(54.97)$ & $344(45.03)$ & 0.051 \\
\hline$\chi^{2}$ & & & 0.919 & & & & \\
\hline$P$ & & & 0.632 & & & & \\
\hline \multicolumn{8}{|l|}{ Han } \\
\hline Male & 268 & $93(34.70)$ & $140(52.24)$ & $35(13.06)$ & $326(60.82)$ & $210(39.18)$ & 0.116 \\
\hline Female & 378 & $137(36.25)$ & $181(47.88)$ & $60(15.87)$ & 455 (60.19) & $301(39.81)$ & 0.987 \\
\hline$\chi^{2}$ & & & 1.547 & & & & \\
\hline$P$ & & & 0.461 & & & & \\
\hline
\end{tabular}

HWE: Hardy-Weinberg equilibrium. The genotype distribution between the two groups was analyzed by the chi-square test. The Hardy-Weinberg equilibrium was analyzed by the chi-square test of the goodness of fit.

(GIH); 55.34\% in Han Chinese in Beijing, China (CHB); $57.56 \%$ in Bengali from Bangladesh (BEB); $64.29 \%$ in Southern Han Chinese (CHS); and 68.82\% in Chinese Dai in Xishuangbanna, China (CDX). In the present study, we found that the Maonan ethnic group had lower rs4810479C allele frequency than the Han ethnic group $(55.61 \%$ vs. $60.45 \%, P<0.05)$. The genotype distribution of the PLTP rs4810479 SNP in the present study was also different between the two ethnic groups $(P<0.05)$, but there was no significant difference in the genotype and allele frequencies between males and females in both populations. These findings suggest that the PLTP rs4810479 SNP may have a racial/ethnic specificity.

The association between the PLTP rs4810479 SNP and serum lipid concentrations in different racial/ethnic groups is still largely unclear. In a previous GWAS, Musunuru et al. 
TABLE 3: Comparison of the genotypes and serum lipid levels in the Maonan and Han populations.

\begin{tabular}{|c|c|c|c|c|c|c|c|c|}
\hline Group/genotype & $n$ & $\mathrm{TC}(\mathrm{mmol} / \mathrm{L})$ & TG $(\mathrm{mmol} / \mathrm{L})$ & HDL-C $(\mathrm{mmol} / \mathrm{L})$ & LDL-C (mmol/L) & ApoA1 (g/L) & ApoB (g/L) & ApoA1/ApoB \\
\hline Maonan & 633 & & & & & & & \\
\hline $\mathrm{CC}$ & 184 & $5.00 \pm 0.96$ & $1.22(0.90)$ & $1.61 \pm 0.39$ & $2.91 \pm 0.83$ & $1.39 \pm 0.23$ & $0.89 \pm 0.19$ & $1.64 \pm 0.55$ \\
\hline CT & 336 & $5.02 \pm 0.97$ & $1.29(0.87)$ & $1.65 \pm 0.40$ & $2.90 \pm 0.79$ & $1.41 \pm 0.24$ & $0.88 \pm 0.19$ & $1.68 \pm 0.49$ \\
\hline TT & 113 & $4.89 \pm 0.96$ & $1.35(0.86)$ & $1.53 \pm 0.34$ & $2.79 \pm 0.81$ & $1.34 \pm 0.22$ & $0.86 \pm 0.19$ & $1.62 \pm 0.42$ \\
\hline$F$ & & 0.779 & 2.052 & 3.281 & 1.366 & 2.756 & 1.173 & 0.329 \\
\hline$P$ & & 0.459 & 0.358 & 0.038 & 0.256 & 0.064 & 0.310 & 0.720 \\
\hline $\mathrm{CC} / \mathrm{CT}$ & 520 & $5.01 \pm 0.97$ & $1.26(0.88)$ & $1.64 \pm 0.40$ & $2.91 \pm 0.81$ & $1.40 \pm 0.24$ & $0.89 \pm 0.19$ & $1.67 \pm 0.51$ \\
\hline$F$ & & 1.509 & -1.389 & 4.844 & 2.712 & 3.721 & 2.109 & 0.024 \\
\hline$P$ & & 0.220 & 0.165 & 0.028 & 0.100 & 0.054 & 0.147 & 0.876 \\
\hline Han & 646 & & & & & & & \\
\hline $\mathrm{CC}$ & 230 & $4.83 \pm 0.88$ & $1.11(0.47)$ & $1.88 \pm 0.44$ & $2.78 \pm 0.59$ & $1.39 \pm 0.22$ & $0.87 \pm 0.20$ & $1.67 \pm 0.44$ \\
\hline CT & 321 & $4.94 \pm 0.92$ & $1.12(0.74)$ & $1.79 \pm 0.41$ & $2.85 \pm 0.73$ & $1.36 \pm 0.23$ & $0.87 \pm 0.19$ & $1.65 \pm 0.46$ \\
\hline TT & 95 & $4.98 \pm 1.04$ & $0.98(0.87)$ & $1.85 \pm 0.41$ & $2.97 \pm 0.83$ & $1.38 \pm 0.23$ & $0.88 \pm 0.21$ & $1.65 \pm 0.50$ \\
\hline$F$ & & 1.409 & 3.369 & 2.719 & 2.710 & 1.059 & 0.394 & 0.070 \\
\hline$P$ & & 0.245 & 0.186 & 0.067 & 0.067 & 0.347 & 0.674 & 0.933 \\
\hline $\mathrm{CC} / \mathrm{CT}$ & 551 & $4.89 \pm 0.91$ & $1.11(0.62)$ & $1.82 \pm 0.42$ & $2.82 \pm 0.68$ & $1.38 \pm 0.23$ & $0.87 \pm 0.20$ & $1.66 \pm 0.45$ \\
\hline$F$ & & 0.774 & -1.578 & 1.132 & 3.615 & 0.457 & 0.493 & 0.034 \\
\hline$P$ & & 0.379 & 0.144 & 0.288 & 0.058 & 0.499 & 0.483 & 0.853 \\
\hline
\end{tabular}

TC: total cholesterol; TG: triglyceride; HDL-C: high-density lipoprotein cholesterol; LDL-C: low-density lipoprotein cholesterol; ApoA1: apolipoprotein A1; ApoB: apolipoprotein B; ApoA1/ApoB: the ratio of apolipoprotein A1 to apolipoprotein B. The value of TG was presented as median (interquartile range); the difference between the genotypes was determined by the Wilcoxon-Mann-Whitney test.

TABLE 4: Comparison of the genotypes and serum lipid levels between males and females in the Maonan and Han populations.

\begin{tabular}{|c|c|c|c|c|c|c|c|c|}
\hline Ethnic/genotype & $n$ & $\mathrm{TC}(\mathrm{mmol} / \mathrm{L})$ & $\mathrm{TG}(\mathrm{mmol} / \mathrm{L})$ & HDL-C $(\mathrm{mmol} / \mathrm{L})$ & LDL-C (mmol/L) & ApoA1 (g/L) & ApoB (g/L) & ApoA1/ApoB \\
\hline \multicolumn{9}{|l|}{ Maonan/male } \\
\hline $\mathrm{CC} / \mathrm{CT}$ & 206 & $4.90 \pm 0.89$ & $1.33(0.98)$ & $1.59 \pm 0.40$ & $2.82 \pm 0.79$ & $1.39 \pm 0.27$ & $0.88 \pm 0.18$ & $1.67 \pm 0.60$ \\
\hline TT & 45 & $4.90 \pm 0.94$ & $1.53(1.52)$ & $1.43 \pm 0.30$ & $2.74 \pm 0.81$ & $1.33 \pm 0.21$ & $0.89 \pm 0.18$ & $1.55 \pm 0.39$ \\
\hline$F$ & & 0.002 & -1.350 & 4.244 & 0.860 & 0.718 & 0.106 & 0.608 \\
\hline$P$ & & 0.962 & 0.177 & 0.040 & 0.355 & 0.398 & 0.745 & 0.436 \\
\hline \multicolumn{9}{|l|}{ Maonan/female } \\
\hline $\mathrm{CC} / \mathrm{CT}$ & 314 & $5.09 \pm 1.01$ & $1.21(0.82)$ & $1.67 \pm 0.40$ & $2.96 \pm 0.81$ & $1.41 \pm 0.21$ & $0.89 \pm 0.19$ & $1.66 \pm 0.46$ \\
\hline TT & 68 & $4.88 \pm 0.99$ & $1.21(0.61)$ & $1.59 \pm 0.36$ & $2.83 \pm 0.81$ & $1.35 \pm 0.22$ & $0.84 \pm 0.19$ & $1.67 \pm 0.43$ \\
\hline$F$ & & 1.799 & -0.800 & 1.639 & 1.302 & 3.341 & 3.365 & 0.135 \\
\hline$P$ & & 0.181 & 0.424 & 0.201 & 0.255 & 0.068 & 0.067 & 0.714 \\
\hline \multicolumn{9}{|l|}{ Han/male } \\
\hline $\mathrm{CC} / \mathrm{CT}$ & 233 & $4.95 \pm 0.86$ & $1.16(0.78)$ & $1.78 \pm 0.44$ & $2.85 \pm 0.63$ & $1.37 \pm 0.26$ & $0.92 \pm 0.21$ & $1.58 \pm 0.48$ \\
\hline TT & 35 & $5.31 \pm 1.06$ & $1.09(0.61)$ & $1.84 \pm 0.43$ & $3.22 \pm 0.87$ & $1.41 \pm 0.27$ & $0.98 \pm 0.22$ & $1.52 \pm 0.53$ \\
\hline$F$ & & 5.189 & -0.974 & 0.835 & 9.517 & 1.250 & 2.797 & 0.179 \\
\hline$P$ & & 0.024 & 0.330 & 0.362 & 0.002 & 0.264 & 0.096 & 0.672 \\
\hline \multicolumn{9}{|l|}{ Han/female } \\
\hline $\mathrm{CC} / \mathrm{CT}$ & 318 & $4.84 \pm 0.93$ & $1.07(0.54)$ & $1.86 \pm 0.41$ & $2.80 \pm 0.71$ & $1.38 \pm 0.21$ & $0.83 \pm 0.18$ & $1.72 \pm 0.41$ \\
\hline $\mathrm{TT}$ & 60 & $4.79 \pm 0.98$ & $0.96(0.93)$ & $1.86 \pm 0.40$ & $2.83 \pm 0.77$ & $1.36 \pm 0.21$ & $0.83 \pm 0.18$ & $1.73 \pm 0.46$ \\
\hline$F$ & & 0.046 & -1.143 & 0.396 & 0.167 & 0.005 & 0.128 & 0.262 \\
\hline$P$ & & 0.831 & 0.253 & 0.529 & 0.683 & 0.946 & 0.721 & 0.609 \\
\hline
\end{tabular}

TC: total cholesterol; TG: triglyceride; HDL-C: high-density lipoprotein cholesterol; LDL-C: low-density lipoprotein cholesterol; ApoA1: apolipoprotein A1; ApoB: apolipoprotein B; ApoA1/ApoB: the ratio of apolipoprotein A1 to apolipoprotein B. The value of triglyceride was presented as median (interquartile range); the difference among the genotypes was determined by the Wilcoxon-Mann-Whitney test.

[36] prompted that the PLTP rs4810479 SNP was associated with HDL-C concentrations in European populations. In the present study, we noted that the PLTP rs4810479 SNP was significantly associated with several serum lipid phenotypes in the Maonan and Han populations. Subgroup analyses of serum lipid profiles according to sex showed that the $\mathrm{C}$ allele carriers had higher HDL-C concentrations in Maonan males and lower TC and LDL-C concentrations in Han males than the $\mathrm{C}$ allele noncarriers. These results indicate that there might be a race- and/or sex-specific association between the PLTP rs4810479 SNP and serum lipid traits in our study ethnic groups.

It is well known that serum lipid concentrations are also affected by many environmental risk factors such as population features, life style, diet structure, and physical inactivity [37]. In the current study, we also found that serum 
TABle 5: Relationship between serum lipid parameters and relative factors in the Maonan and Han populations.

\begin{tabular}{|c|c|c|c|c|c|c|}
\hline Lipid & Risk factor & $\mathrm{B}$ & Std. error & Beta & $t$ & $P$ \\
\hline \multicolumn{7}{|c|}{ Maonan and Han } \\
\hline \multirow{4}{*}{$\mathrm{TC}$} & Waist circumference & 0.018 & 0.003 & 0.162 & 5.487 & $<0.001$ \\
\hline & Age & 0.007 & 0.002 & 0.106 & 3.688 & $<0.001$ \\
\hline & Height & -0.010 & 0.003 & -0.088 & -2.987 & 0.003 \\
\hline & Diastolic blood pressure & 0.005 & 0.002 & 0.066 & 2.293 & 0.022 \\
\hline \multirow{4}{*}{ TG } & Waist circumference & 0.038 & 0.003 & 0.302 & 11.155 & $<0.001$ \\
\hline & Alcohol consumption & 0.298 & 0.073 & 0.109 & 4.106 & $<0.001$ \\
\hline & Glucose & 0.069 & 0.022 & 0.082 & 3.142 & 0.002 \\
\hline & Diastolic blood pressure & 0.008 & 0.003 & 0.081 & 3.024 & 0.003 \\
\hline \multirow{8}{*}{ HDL-C } & Waist circumference & -0.012 & 0.002 & -0.245 & -6.804 & $<0.001$ \\
\hline & Ethnic group & 0.189 & 0.022 & 0.225 & 8.779 & $<0.001$ \\
\hline & Alcohol consumption & 0.135 & 0.033 & 0.128 & 4.045 . & $<0.001$ \\
\hline & Gender & 0.123 & 0.031 & 0.143 & 3.996 & $<0.001$ \\
\hline & Age & 0.003 & 0.001 & 0.090 & 3.163 & 0.002 \\
\hline & Body mass index & -0.010 & 0.004 & -0.082 & -2.338 & 0.020 \\
\hline & Pulse pressure & -0.002 & 0.001 & -0.060 & -2.123 & 0.034 \\
\hline & Cigarette smoking & 0.067 & 0.033 & 0.067 & 1.987 & 0,047 \\
\hline \multirow{3}{*}{ LDL-C } & Waist circumference & 0.017 & 0.003 & 0.199 & 6.917 & $<0.001$ \\
\hline & Age & 0.007 & 0.001 & 0.125 & 4.499 & $<0.001$ \\
\hline & Height & -0.009 & 0.003 & -0.096 & -3.291 & 0.001 \\
\hline \multirow{5}{*}{ ApoA1 } & Waist circumference & -0.003 & 0.001 & -0.103 & -2.453 & 0.014 \\
\hline & Alcohol consumption & 0.152 & 0.019 & 0.264 & 7.792 & $<0.001$ \\
\hline & Gender & 0.076 & 0.018 & 0.162 & 4.191 & $<0.001$ \\
\hline & Cigarette smoking & 0.064 & 0.019 & 0.117 & 3.311 & 0.001 \\
\hline & Weight & -0.003 & 0.001 & -0.127 & -2.831 & 0.005 \\
\hline \multirow{4}{*}{ ApoB } & Waist circumference & 0.006 & 0.001 & 0.280 & 10.346 & $<0.001$ \\
\hline & Age & 0.002 & 0.000 & 0.136 & 4.914 & $<0.001$ \\
\hline & Diastolic blood pressure & 0.001 & 0.000 & 0.073 & 2.624 & 0.009 \\
\hline & Glucose & 0.010 & 0.004 & 0.065 & 2.399 & 0.017 \\
\hline \multirow{7}{*}{ ApoA1/ApoB } & Waist circumference & -0.014 & 0.002 & -0.244 & -6.668 & $<0.001$ \\
\hline & Alcohol consumption & 0.214 & 0.038 & 0.180 & 5.552 & $<0.001$ \\
\hline & Gender & 0.161 & 0.035 & 0.166 & 4.532 & $<0.001$ \\
\hline & Age & -0.003 & 0.001 & -0.082 & -3.065 & 0.002 \\
\hline & Body mass index & -0.015 & 0.005 & -0.107 & -2.998 & 0.003 \\
\hline & Cigarette smoking & 0.089 & 0.039 & 0.079 & 2.294 & 0.022 \\
\hline & Glucose & -0.022 & 0.010 & -0.060 & -2.234 & 0.026 \\
\hline \multicolumn{7}{|l|}{ Maonan } \\
\hline \multirow{3}{*}{$\mathrm{TC}$} & Waist circumference & 0.025 & 0.004 & 0.233 & 5.810 & $<0.001$ \\
\hline & Age & 0.011 & 0.003 & 0.165 & 4.291 & $<0.001$ \\
\hline & Gender & 0.285 & 0.079 & 0.144 & 3.601 & $<0.001$ \\
\hline \multirow{4}{*}{ TG } & Waist circumference & 0.038 & 0.004 & 0.345 & 9.209 & $<0.001$ \\
\hline & Glucose & 0.084 & 0.029 & 0.106 & 2.904 & 0.004 \\
\hline & Diastolic blood pressure & 0.007 & 0.003 & 0.089 & 2.367 & 0.018 \\
\hline & Alcohol consumption & 0.201 & 0.090 & 0.083 & 2.227 & 0.026 \\
\hline \multirow{4}{*}{ HDL-C } & Waist circumference & -0.014 & 0.002 & -0.333 & -8.635 & $<0.001$ \\
\hline & Genotype & -0.112 & 0.038 & -0.110 & -2.971 & 0.003 \\
\hline & Alcohol consumption & 0.150 & 0.044 & 0.157 & 3.444 & 0.001 \\
\hline & Gender & 0.094 & 0.038 & 0.117 & 2.503 & 0.013 \\
\hline \multirow{3}{*}{ LDL-C } & Waist circumference & 0.023 & 0.004 & 0.262 & 6.566 & $<0.001$ \\
\hline & Gender & 0263 & 0.066 & 0.160 & 4.018 & $<0.001$ \\
\hline & Age & 0.009 & 0.002 & 0.152 & 3.981 & $<0.001$ \\
\hline \multirow{5}{*}{ ApoA1 } & Waist circumference & -0.006 & 0.001 & -0.219 & -5.605 & $<0.001$ \\
\hline & Alcohol consumption & 0.140 & 0.027 & 0.245 & 5.132 & $<0.001$ \\
\hline & Gender & 0.101 & 0.026 & 0.211 & 3.909 & $<0.001$ \\
\hline & Cigarette smoking & 0.082 & 0.029 & 0.143 & 2.829 & 0.005 \\
\hline & Genotype & -0.054 & 0.023 & -0.089 & -2.361 & 0.019 \\
\hline
\end{tabular}


TABLE 5: Continued.

\begin{tabular}{|c|c|c|c|c|c|c|}
\hline Lipid & Risk factor & B & Std. error & Beta & $t$ & $P$ \\
\hline \multirow{3}{*}{ ApoB } & Waist circumference & 0.008 & 0.001 & 0.385 & 10.056 & $<0.001$ \\
\hline & Age & 0.002 & 0.000 & 0.181 & 4.945 & $<0.001$ \\
\hline & Gender & 0.042 & 0.015 & 0.109 & 2.866 & 0.040 \\
\hline \multirow{3}{*}{ ApoA1/ApoB } & Waist circumference & -0.022 & 0.002 & -0.390 & -10.608 & $<0.001$ \\
\hline & Alcohol consumption & 0.224 & 0.045 & 0.184 & 4.499 & $<0.001$ \\
\hline & Age & -0.004 & 0.001 & -0.110 & -3.023 & 0.003 \\
\hline \multicolumn{7}{|l|}{ Han } \\
\hline \multirow{3}{*}{ TC } & Diastolic blood pressure & 0.011 & 0.003 & 0.128 & 3.153 & 0.002 \\
\hline & Age & 0.006 & 0.003 & 0.098 & 2.478 & 0.013 \\
\hline & Body mass index & 0.024 & 0.012 & 0.085 & 2.126 & 0.034 \\
\hline \multirow{3}{*}{ TG } & Waist circumference & 0.039 & 0.006 & 0.266 & 6.837 & $<0.001$ \\
\hline & Alcohol consumption & 0.370 & 0.114 & 0.123 & 3.249 & 0.001 \\
\hline & Diastolic blood pressure & 0.008 & 0.004 & 0.077 & 1.995 & 0.046 \\
\hline \multirow[b]{2}{*}{ HDL-C } & Waist circumference & -0.011 & 0.003 & -0.215 & -3.871 & $<0.001$ \\
\hline & Body mass index & -0.016 & 0.007 & -0.120 & -2.159 & 0.031 \\
\hline \multirow{2}{*}{ LDL-C } & Age & 0.007 & 0.002 & 0.155 & 4.003 & $<0.001$ \\
\hline & Body mass index & 0.031 & 0.008 & 0.140 & 3.608 & $<0.001$ \\
\hline \multirow{2}{*}{ ApoA1 } & Alcohol consumption & 0.168 & 0.023 & 0.288 & 7.189 & $<0.001$ \\
\hline & Weight & -0.007 & 0.001 & -0.282 & -7.052 & $<0.001$ \\
\hline \multirow{5}{*}{ ApoB } & Waist circumference & 0.005 & 0.001 & 0.189 & 4.863 & $<0.001$ \\
\hline & Gender & -0.100 & 0.018 & -0.248 & -5.554 & $<0.001$ \\
\hline & Diastolic blood pressure & 0.003 & 0.001 & 0.142 & 3.766 & $<0.001$ \\
\hline & Glucose & 0.018 & 0.005 & 0.121 & 3.286 & 0.001 \\
\hline & Height & -0.003 & 0.001 & -0.121 & -2.675 & 0.008 \\
\hline \multirow{5}{*}{ ApoA1/ApoB } & Body mass index & -0.039 & 0.005 & -0.275 & -7.287 & $<0.001$ \\
\hline & Glucose & -0.042 & 0.013 & -0.124 & -3.360 & $<0.001$ \\
\hline & Gender & 0.213 & 0.040 & 0.232 & 5.359 & 0.001 \\
\hline & Alcohol consumption & 0.231 & 0.051 & 0.200 & 4.540 & $<0.001$ \\
\hline & Diastolic blood pressure & -0.004 & 0.002 & -0.105 & -2.824 & 0.005 \\
\hline
\end{tabular}

TC: total cholesterol; TG: triglyceride; HDL-C: high-density lipoprotein cholesterol; LDL-C: low-density lipoprotein cholesterol; ApoA1: apolipoprotein A1; ApoB: apolipoprotein B; ApoA1/ApoB: the ratio of apolipoprotein A1 to apolipoprotein B; B: unstandardized coefficient; Beta: standardized coefficient.

TABLE 6: Relationship between serum lipid parameters and relative factors in the males and females of the Han and Maonan populations.

\begin{tabular}{|c|c|c|c|c|c|c|}
\hline Lipid & Risk factor & $\mathrm{B}$ & Std. error & Beta & $t$ & $P$ \\
\hline \multicolumn{7}{|c|}{ Maonan/male } \\
\hline \multirow{4}{*}{ TC } & Weight & 0.029 & 0.005 & 0.332 & 5.320 & $<0.001$ \\
\hline & Glucose & 0.103 & 0.041 & 0.151 & 2.527 & 0.012 \\
\hline & Pulse pressure & -0.010 & 0.003 & -0.190 & -2.831 & 0.005 \\
\hline & Age & 0.010 & 0.004 & 0.161 & 2.276 & 0.024 \\
\hline \multirow{3}{*}{ TG } & Waist circumference & 0.054 & 0.008 & 0.401 & 6.975 & $<0.001$ \\
\hline & Glucose & 0.179 & 0.054 & 0.191 & 3.321 & 0.001 \\
\hline & Alcohol consumption & 0.365 & 0.142 & 0.148 & 2.574 & 0.011 \\
\hline \multirow{3}{*}{ HDL-C } & Waist circumference & -0.015 & 0.002 & -0.353 & -6.195 & $<0.001$ \\
\hline & Alcohol consumption & 0.170 & 0.044 & 0.221 & 3.883 & $<0.001$ \\
\hline & Genotype & -0.134 & 0.057 & -0.133 & -2.348 & 0.020 \\
\hline \multirow{4}{*}{ LDL-C } & Weight & 0.024 & 0.005 & 0.310 & 4.954 & $<0.001$ \\
\hline & Alcohol consumption & -0.203 & 0.095 & -0.128 & -2.123 & 0.035 \\
\hline & Pulse pressure & -0.010 & 0.003 & -0.212 & -3.143 & 0.002 \\
\hline & Age & 0.009 & 0.004 & 0.164 & 2.297 & 0.022 \\
\hline \multirow{3}{*}{ ApoA1 } & Alcohol consumption & 0.153 & 0.031 & 0.293 & 4.898 & $<0.001$ \\
\hline & Waist circumference & -0.005 & 0.002 & -0.186 & -3.231 & 0.001 \\
\hline & Cigarette smoking & 0.085 & 0.031 & 0.162 & 2.713 & 0.007 \\
\hline
\end{tabular}


TABle 6: Continued.

\begin{tabular}{|c|c|c|c|c|c|c|}
\hline Lipid & Risk factor & $\mathrm{B}$ & Std. error & Beta & $t$ & $P$ \\
\hline \multirow{5}{*}{ ApoB } & Waist circumference & 0.005 & 0.002 & 0.234 & 2.342 & 0.020 \\
\hline & Glucose & 0.025 & 0.008 & 0.183 & 3.254 & 0.001 \\
\hline & Pulse pressure & -0.002 & 0.001 & -0.200 & -3.164 & 0.002 \\
\hline & Age & 0.002 & 0.001 & 0.179 & 2.638 & 0.009 \\
\hline & Weight & 0.004 & 0.002 & 0,242 & 2.319 & 0.021 \\
\hline \multirow{3}{*}{ ApoA1/ApoB } & Waist circumference & -0.021 & 0.004 & -0.343 & -6.021 & $<0.001$ \\
\hline & Alcohol consumption & 0.301 & 0.064 & 0.267 & 4.691 & $<0.001$ \\
\hline & Systolic blood pressure & 0.003 & 0.001 & 0.131 & 2.313 & 0.022 \\
\hline \multicolumn{7}{|l|}{ Maonan/female } \\
\hline \multirow{3}{*}{ 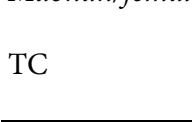 } & Age & 0.019 & 0.004 & 0.271 & 5.469 & $<0.001$ \\
\hline & Waist circumference & 0.024 & 0.006 & 0.197 & 3.997 & $<0.001$ \\
\hline & Glucose & -0.085 & 0.042 & -0.102 & -2.035 & 0.043 \\
\hline \multirow{2}{*}{ TG } & Waist circumference & 0.033 & 0.004 & 0.357 & 7.518 & $<0.001$ \\
\hline & Age & 0.008 & 0.003 & 0.143 & 3.009 & 0.003 \\
\hline \multirow{2}{*}{ HDL-C } & Waist circumference & -0.014 & 0.002 & -0.288 & -5.861 & $<0.001$ \\
\hline & Genotype & -0.100 & 0.050 & -0.098 & -1.992 & 0.047 \\
\hline \multirow{3}{*}{ LDL-C } & Age & 0.013 & 0.003 & 0.235 & 4.870 & $<0.001$ \\
\hline & Waist circumference & 0.020 & 0.005 & 0.209 & 4.264 & $<0.001$ \\
\hline & Alcohol consumption & 0.619 & 0.297 & 0.102 & 2.086 & 0.038 \\
\hline \multirow{2}{*}{ AopA1 } & Waist circumference & -0.006 & 0.001 & -0.221 & -4.423 & $<0.001$ \\
\hline & Genotype & -0.067 & 0.028 & -0.120 & -2.406 & 0.017 \\
\hline \multirow{3}{*}{ ApoB } & Waist circumference & 0.008 & 0.001 & 0.351 & 7.481 & $<0.001$ \\
\hline & Age & 0.004 & 0.001 & 0.276 & 5.847 & $<0.001$ \\
\hline & Glucose & -0.016 & 0.008 & -0.097 & -2.027 & 0.043 \\
\hline \multirow{3}{*}{ ApoA1/ApoB } & Waist circumference & -0.020 & 0.003 & -0.377 & -7.697 & $<0.001$ \\
\hline & Systolic blood pressure & -0.003 & 0.001 & -0.168 & -3.545 & $<0.001$ \\
\hline & Height & 0.010 & 0.004 & 0.139 & 2.907 & 0.004 \\
\hline \multicolumn{7}{|l|}{ Han/male } \\
\hline \multirow{2}{*}{$\mathrm{TC}$} & Diastolic blood pressure & 0.014 & 0.005 & 0.187 & 3.121 & 0.005 \\
\hline & Genotype & 0.360 & 0.159 & 0.136 & 2.269 & 0.024 \\
\hline \multirow{2}{*}{ TG } & Waist circumference & 0.051 & 0.010 & 0.290 & 4.939 & $<0.001$ \\
\hline & Alcohol consumption & 0.380 & 0.165 & 0.135 & 2.302 & 0.022 \\
\hline \multirow{2}{*}{ HDL-C } & Weight & -0.016 & 0.003 & -0.299 & -4.959 & $<0.001$ \\
\hline & Alcohol consumption & 0.133 & 0.053 & 0.151 & 2.504 & 0.013 \\
\hline \multirow{3}{*}{ LDL-C } & Genotype & 0.372 & 0.119 & 0.186 & 3.136 & 0.002 \\
\hline & Body mass index & 0.029 & 0.012 & 0.149 & 2.507 & 0.013 \\
\hline & Glucose & 0.060 & 0.027 & 0.132 & 2.230 & 0.027 \\
\hline \multirow{2}{*}{ ApoA1 } & Alcohol consumption & 0.193 & 0.030 & 0.368 & 6.383 & $<0.001$ \\
\hline & Weight & -0.008 & 0.002 & -0.276 & -4.784 & $<0.001$ \\
\hline \multirow{3}{*}{ ApoB } & Body mass index & 0.012 & 0.004 & 0.197 & 3.322 & 0.001 \\
\hline & Glucose & 0.028 & 0.008 & 0.199 & 3.426 & 0.001 \\
\hline & Diastolic blood pressure & 0.003 & 0.001 & 0.159 & 2.681 & 0.008 \\
\hline \multirow{3}{*}{ ApoA1/ApoB } & Weight & -0.019 & 0.003 & -0.337 & -5.821 & $<0.001$ \\
\hline & Alcohol consumption & 0.234 & 0.058 & 0.239 & 4.050 & $<0.001$ \\
\hline & Glucose & -0.051 & 0.019 & -0.156 & -2.717 & 0.007 \\
\hline Han/female & & & & & & \\
\hline $\mathrm{TC}$ & Age & 0.014 & 0.004 & 0.208 & 3.986 & $<0.001$ \\
\hline $1 \mathrm{C}$ & Height & -0.020 & 0.008 & -0.130 & -2.505 & 0.013 \\
\hline & Waist circumference & 0.029 & 0.006 & 0.234 & 4.573 & $<0.001$ \\
\hline TG & Diastolic blood pressure & 0.014 & 0.005 & 0.145 & 2.825 & 0.005 \\
\hline & Waist circumference & -0.009 & 0.004 & -0.184 & -2.259 & 0.024 \\
\hline HDL-C & Body mass index & -0.023 & 0.011 & -0.169 & -2.077 & 0.039 \\
\hline IDI_C & Age & 0.014 & 0.003 & 0.265 & 5.363 & $<0.001$ \\
\hline LDL-C & Body mass index & 0.027 & 0.012 & 0.112 & 2.275 & 0.023 \\
\hline ApoA1 & Body mass index & -0.015 & 0.003 & -0.220 & -4.364 & $<0.001$ \\
\hline
\end{tabular}


TABLE 6: Continued.

\begin{tabular}{|c|c|c|c|c|c|c|}
\hline Lipid & Risk factor & $\mathrm{B}$ & Std. error & Beta & $t$ & $P$ \\
\hline \multirow{3}{*}{ ApoB } & Age & 0.004 & 0.001 & 0.286 & 5.829 & $<0.001$ \\
\hline & Body mass index & 0.012 & 0.003 & 0.205 & 4.241 & $<0.001$ \\
\hline & Cigarette smoking & -0.156 & 0.058 & -0.130 & -2.663 & 0.008 \\
\hline \multirow{3}{*}{ ApoA1/ApoB } & Age & -0.006 & 0.001 & -0.209 & -4.279 & $<0.001$ \\
\hline & Cigarette smoking & 0.413 & 0.133 & -0.151 & 3.096 & 0.002 \\
\hline & Body mass index & -0.040 & 0.007 & -0.287 & -5.970 & $<0.001$ \\
\hline
\end{tabular}

TC: total cholesterol; TG: triglyceride; HDL-C: high-density lipoprotein cholesterol; LDL-C: low-density lipoprotein cholesterol; ApoA1: apolipoprotein A1; ApoB: apolipoprotein B; ApoA1/ApoB: the ratio of apolipoprotein A1 to apolipoprotein B; B: unstandardized coefficient; Beta: standardized coefficient. The correlation among serum lipid parameters and the genotypes and several environmental factors was determined by multivariable linear regression analyses with stepwise modeling.

lipid concentrations were associated with several environmental risk factors in both ethnic groups. In a previous research study, we found that the intakes of total dietary fat, cholesterol, and energy were higher in Maonan than in Han ethnic groups [30]. The difference in living environment, eating habits, life style, and genetic background between the two populations may be the main cause of different serum lipid concentrations. Rice, corn, and other carbohydraterich foods are the daily staple foods of the Maonan people. They are also good at making various complementary foods with rice. They like to eat spicy and acidic foods that contain a lot of oil and salt. The intake of a large amount of carbohydrate, oil, and salt can increase the waist circumference and blood pressure in the Maonan people. Several studies have shown that long-term high-salt diet is an important risk factor to affect blood pressure levels [38, 39]. A meta-analysis showed that reduced sodium intake can lower blood pressure levels in people with or without hypertension [40]. In addition, the people of Maonan also like to eat pork, beef, and/or animal offals in a hot pot which is rich in saturated fatty acids. Many previous studies have shown that diet alone can explain the variation in blood lipid levels [41]. Longterm high-saturated fat diets are strongly associated with obesity, hypertension, dyslipidemia, and atherosclerosis $[42,43]$. Therefore, different environmental risk factors such as unhealthy lifestyle and diet structure may further alter the association between genetic variation and blood lipid concentrations in our research populations.

Our work may have some limitations. First, we could not exclude the influence of diet and other environmental risk factors in the statistical analyses. Second, we also could not rule out the effect of asymptomatic diseases. Third, an association between the PLTP rs4810479 SNP and serum lipid concentrations was observed in this study, but many unmeasured factors should be considered including genetic and environmental risk factors. Finally, the sample size in our study populations is a bit small. Therefore, it is necessary to further expand the sample size, especially the gene-gene, gene-environment, and environment-environment interactions on serum lipid parameters to confirm our findings.

\section{Conclusions}

There was a significant difference in the genotype and allele distribution of the PLTP rs4810479 SNP between the Maonan and Han populations. The association between the PLTP rs4810479 SNP and serum lipid parameters was also different between the two nationalities and between males and females. There may be a racial/ethnic- and/or sexspecific association between the PLTP rs4810479 SNP and serum lipid concentrations in our study populations.

\section{Abbreviations}

ANCOVA: Analysis of covariance

Apo: $\quad$ Apolipoprotein

BMI: $\quad$ Body mass index

BPI: Bactericidal/permeability-increasing protein

CAAD: $\quad$ Carotid artery disease

CAD: $\quad$ Coronary artery disease

CETP: Cholesterol ester transfer protein

CVD: Cardiovascular disease

DNA: Deoxyribonucleic acid

GWAS: Genome-wide association study

HDL-C: High-density lipoprotein cholesterol

LBP: $\quad$ Lipopolysaccharide-binding protein

LDL-C: Low-density lipoprotein cholesterol

LPS: $\quad$ Lipopolysaccharide

PCR: $\quad$ Polymerase chain reaction

PLTP: Phospholipid transfer protein

PLTPa: PLTP activity

RFLP: $\quad$ Restriction fragment length polymorphism

SNP: $\quad$ Single-nucleotide polymorphism

TC: $\quad$ Total cholesterol

TG: $\quad$ Triglyceride

UTR: Untranslated region.

\section{Data Availability}

The datasets generated during the present study are not publicly available because detailed genetic information of each participant was included in these materials. 


\section{Disclosure}

There was no role of the funding body in the design of the study and collection, analysis, and interpretation of the data and in writing the manuscript.

\section{Conflicts of Interest}

The authors declare no conflicts of interest.

\section{Authors' Contributions}

F.-H. Z. conceived the study, participated in the design, undertook genotyping, performed the statistical analyses, and drafted the manuscript. R.-X. Y. conceived the study, participated in the design, carried out the epidemiological survey, collected the samples, and helped to draft the manuscript. L.-M. Y., W.-X. L., J.-Z. W., and D.-Z. Y. carried out the epidemiological survey and collected the samples. All authors read and approved the final manuscript.

\section{Acknowledgments}

The authors are grateful for the funding support provided by the National Natural Science Foundation of China (no. 81460169). They also greatly thank all the participants of this study.

\section{References}

[1] G. A. Roth, C. Johnson, A. Abajobir et al., "Global, regional, and national burden of cardiovascular diseases for 10 causes, 1990 to 2015," Journal of the American College of Cardiology, vol. 70, no. 1, pp. 1-25, 2017.

[2] M. J. Chapman, H. N. Ginsberg, P. Amarenco et al., "Triglyceride-rich lipoproteins and high-density lipoprotein cholesterol in patients at high risk of cardiovascular disease: evidence and guidance for management," European Heart Journal, vol. 32, no. 11, pp. 1345-1361, 2011.

[3] B. Lamarche, J.-P. Després, S. Moorjani, B. Cantin, G. R. Dagenais, and P.-J. Lupien, "Triglycerides and HDLcholesterol as risk factors for ischemic heart disease. Results from the Québec cardiovascular study," Atherosclerosis, vol. 119, no. 2, pp. 235-245, 1996.

[4] A. V. Khera, M. Cuchel, M. de la Llera-Moya et al., "Cholesterol efflux capacity, high-density lipoprotein function, and atherosclerosis," New England Journal of Medicine, vol. 364, no. 2, pp. 127-135, 2011.

[5] I. M. Graham, A. L. Catapano, and N. D. Wong, "Current guidelines on prevention with a focus on dyslipidemias," Cardiovascular Diagnosis and Therapy, vol. 7, no. Suppl 1, pp. S4-S10, 2017.

[6] Y. Ruixing, C. Yuming, P. Shangling et al., "Effects of demographic, dietary and other lifestyle factors on the prevalence of hyperlipidemia in Guangxi Hei Yi Zhuang and Han populations," European Journal of Cardiovascular Prevention \& Rehabilitation, vol. 13, no. 6, pp. 977-984, 2006.

[7] S. Kathiresan, O. Melander, D. Anevski et al., "Polymorphisms associated with cholesterol and risk of cardiovascular events," New England Journal of Medicine, vol. 358, no. 12, pp. 1240-1249, 2008.

[8] D. M. Waterworth, S. L. Ricketts, K. Song et al., "Genetic variants influencing circulating lipid levels and risk of coronary artery disease," Arteriosclerosis, Thrombosis, and Vascular Biology, vol. 30, no. 11, pp. 2264-2276, 2010.

[9] N. J. Samani, J. Erdmann, A. S. Hall et al., "Genomewide association analysis of coronary artery disease," New England Journal of Medicine, vol. 357, no. 5, pp. 443-453, 2007.

[10] C. J. Willer, S. Sanna, A. U. Jackson et al., "Newly identified loci that influence lipid concentrations and risk of coronary artery disease," Nature Genetics, vol. 40, no. 2, pp. 161-169, 2008.

[11] T. M. Teslovich, K. Musunuru, A. V. Smith et al., "Biological, clinical and population relevance of 95 loci for blood lipids," Nature, vol. 466, no. 7307, pp. 707-713, 2010.

[12] C. J. Willer, E. M. Schmidt, S. Sengupta et al., "Discovery and refinement of loci associated with lipid levels," Nature Genetics, vol. 45, no. 11, pp. 1274-1283, 2013.

[13] A. Yazdanyar, C. Yeang, and X. C. Jiang, "Role of phospholipid transfer protein in high-density lipoprotein-mediated reverse cholesterol transport," Current Atherosclerosis Reports, vol. 13, no. 3, pp. 244-248, 2011.

[14] X.-C. Jiang, W. Jin, and M. M. Hussain, "The impact of phospholipid transfer protein (PLTP) on lipoprotein metabolism," Nutrition \& Metabolism, vol. 9, no. 1, p. 75, 2012.

[15] X.-C. Jiang, "Impact of phospholipid transfer protein in lipid metabolism and cardiovascular diseases," Advances in Experimental Medicine and Biology, vol. 1276, no. 1, pp. 1-13, 2020.

[16] T. Oka, T. Kujiraoka, M. Ito et al., "Distribution of phospholipid transfer protein in human plasma: presence of two forms of phospholipid transfer protein, one catalytically active and the other inactive," Journal of Lipid Research, vol. 41, no. 10 , pp. 1651-1657, 2000.

[17] J. R. Day, J. J. Albers, C. E. Lofton-Day et al., "Complete cDNA encoding human phospholipid transfer protein from human endothelial cells," Journal of Biological Chemistry, vol. 269, no. 12, pp. 9388-9391, 1994.

[18] D. T. Valenta, N. Ogier, G. Bradshaw et al., "Atheroprotective potential of macrophage-derived phospholipid transfer protein in low-density lipoprotein receptor-deficient mice is overcome by apolipoprotein AI overexpression," Arteriosclerosis, Thrombosis, and Vascular Biology, vol. 26, no. 7, pp. 1572-1578, 2006.

[19] C. M. Desrumaux, P. A. Mak, W. A. Boisvert et al., "Phospholipid transfer protein is present in human atherosclerotic lesions and is expressed by macrophages and foam cells," Journal of Lipid Research, vol. 44, no. 8, pp. 1453-1461, 2003.

[20] K. D. O’Brien, S. Vuletic, T. O. McDonald et al., "Cell-associated and extracellular phospholipid transfer protein in human coronary atherosclerosis," Circulation, vol. 108, no. 3, pp. 270-274, 2003.

[21] T. Tzotzas, C. Desrumaux, and L. Lagrost, "Plasma phospholipid transfer protein (PLTP): review of an emerging cardiometabolic risk factor," Obesity Reviews, vol. 10, no. 4, pp. 403-411, 2009.

[22] R. P. F. Dullaart, M. Vergeer, R. de Vries, P. J. W. H. Kappelle, and G. M. Dallinga-Thie, "Type 2 diabetes mellitus interacts with obesity and common variations in PLTP to affect plasma phospholipid transfer protein activity," Journal of Internal Medicine, vol. 271, no. 5, pp. 490-498, 2012.

[23] J. Huuskonen, V. M. Olkkonen, M. Jauhiainen, and C. Ehnholm, "The impact of phospholipid transfer protein (PLTP) on HDL metabolism," Atherosclerosis, vol. 155, no. 2, pp. 269-281, 2001.

[24] Y. Si, Y. Zhang, X. Chen et al., "Phospholipid transfer protein deficiency in mice impairs macrophage reverse cholesterol 
transport in vivo," Experimental Biology and Medicine, vol. 241, no. 13, pp. 1466-1472, 2016.

[25] G. Song, C. Zong, M. Shao et al., "Phospholipid transfer protein (PLTP) deficiency attenuates high fat diet induced obesity and insulin resistance," Biochimica et Biophysica Acta (BBA)-Molecular and Cell Biology of Lipids, vol. 1864, no. 10, pp. 1305-1313, 2019.

[26] D. S. Kim, A. A. Burt, J. E. Ranchalis et al., "PLTP activity inversely correlates with CAAD: effects of PON1 enzyme activity and genetic variants on PLTP activity," Journal of Lipid Research, vol. 56, no. 7, pp. 1351-1362, 2015.

[27] G. P. Jarvik, R. Rajagopalan, E. A. Rosenthal et al., "Genetic and nongenetic sources of variation in phospholipid transfer protein activity," Journal of Lipid Research, vol. 51, no. 5, pp. 983-990, 2010.

[28] S. Kathiresan, C. J. Willer, G. M. Peloso et al., "Common variants at 30 loci contribute to polygenic dyslipidemia," Nature Genetics, vol. 41, no. 1, pp. 56-65, 2009.

[29] M. Vergeer, S. M. Boekholdt, M. S. Sandhu et al., "Genetic variation at the phospholipid transfer protein locus affects its activity and high-density lipoprotein size and is a novel marker of cardiovascular disease susceptibility," Circulation, vol. 122 , no. 5, pp. 470-477, 2010.

[30] Y. Wang, L. H. Aung, J. Y. Tan et al., "Prevalence of dyslipidemia and its risk factors in the Chinese Maonan and Han populations," International Journal of Clinical and Experimental Pathology, vol. 9, no. 10, pp. 10603-10616, 2016.

[31] L. Qiu, R.-X. Yin, E. Khounphinith, F.-H. Zhang, D.-Z. Yang, and S.-L. Pan, "Association of the APOA1 rs964184 SNP and serum lipid traits in the Chinese Maonan and Han populations," Lipids in Health and Disease, vol. 17, no. 1, p. 105, 2018.

[32] People's Republic of China--United States Cardiovascular and Cardiopulmonary Epidemiology Research Group, "An epidemiological study of cardiovascular and cardiopulmonary disease risk factors in four populations in the People's Republic of China. Baseline report from the P.R.C.-U.S.A. Collaborative study. People's Republic of China--United States cardiovascular and cardiopulmonary epidemiology research group," Circulation, vol. 85, no. 3, pp. 1083-1096, 1992.

[33] L. Miao, R.-X. Yin, S.-L. Pan, S. Yang, D.-Z. Yang, and W.-X. Lin, "Association between the MVK and MMAB polymorphisms and serum lipid levels," Oncotarget, vol. 8, no. 41, pp. 70378-70393, 2017.

[34] Q.-H. Zhang, R.-X. Yin, H. Gao et al., "Association of the SPTLC3 rs364585 polymorphism and serum lipid profiles in two Chinese ethnic groups," Lipids in Health and Disease, vol. 16, no. 1, p. 1, 2017.

[35] L. Miao, R.-X. Yin, S.-L. Pan, S. Yang, D.-Z. Yang, and W.-X. Lin, "BCL3-PVRL2-TOMM40 SNPs, gene-gene and gene-environment interactions on dyslipidemia," Scientific Reports, vol. 8, no. 1, p. 6189, 2018.

[36] K. Musunuru, S. P. Romaine, G. Lettre et al., "Multi-ethnic analysis of lipid-associated loci: the NHLBI CARe project," PLoS One, vol. 7, no. 5, Article ID e36473, 2012.

[37] Y. Ruixing, F. Qiming, Y. Dezhai et al., "Comparison of demography, diet, lifestyle, and serum lipid levels between the Guangxi Bai Ku Yao and Han populations," Journal of Lipid Research, vol. 48, no. 12, pp. 2673-2681, 2007.

[38] K. J. Aaron and P. W. Sanders, "Role of dietary salt and potassium intake in cardiovascular health and disease: a review of the evidence," Mayo Clinic Proceedings, vol. 88, no. 9, pp. 987-995, 2013.
[39] P. Rust and C. Ekmekcioglu, "Impact of salt intake on the pathogenesis and treatment of hypertension," Advances in Experimental Medicine and Biology, vol. 956, no. 1, pp. 61-84, 2017.

[40] N. J. Aburto, A. Ziolkovska, L. Hooper, P. Elliott, F. P. Cappuccio, and J. J. Meerpohl, "Effect of lower sodium intake on health: systematic review and meta-analyses," BMJ, vol. 346, no. apr03 3, p. f1326, 2013.

[41] Y. Joffe, M. Collins, and J. Goedecke, "The relationship between dietary fatty acids and inflammatory genes on the obese phenotype and serum lipids," Nutrients, vol. 5, no. 5, pp. 1672-1705, 2013.

[42] S. Chiu, P. T. Williams, and R. M. Krauss, "Effects of a very high saturated fat diet on LDL particles in adults with atherogenic dyslipidemia: A randomized controlled trial," PLoS One, vol. 12, no. 1, Article ID e0170664, 2017.

[43] A. D. von Frankenberg, A. Marina, X. Song, H. S. Callahan, M. Kratz, and K. M. Utzschneider, "A high-fat, high-saturated fat diet decreases insulin sensitivity without changing intraabdominal fat in weight-stable overweight and obese adults," European Journal of Nutrition, vol. 56, no. 1, pp. 431-443, 2017. 